\section{UWB Doublet Generation in an Integrated Semiconductor Optical Amplifier Mach-Zehnder Interferometer}

ABSTRACT

In this papur, we propose and experimentally demonstrate a novel technique to generate ultrawideband (UWB) doublet pulses by exploiting the cross-phase modulation (XPM) in a semiconductor optical amplifier (SOA). The key component in the proposed system consists on an integrated SOA Mach-Zehnder interferometer (MZ) pumped with a Gaussian pulse modulated optical carrier. The transfer function of the nonlinear conversio Keywords: optical pulse generation, ultrawideband technology, radio over fiber, microwave photonics.

\section{INTRODUCTION}

Ultra-wideband (UWB) technology is considered an interesting technology for its application in wireles communications, sensor networks, location and radar systems. The US Federal Communications Commissio FCC) has regulated the definition of UWB signal which has a spectral bandwidth greater than $500 \mathrm{MHz}$ or a fractional bandwidth greater than $20 \%$ with a power spectral density lower than $-41.3 \mathrm{dBm} / \mathrm{MHz}$ withi frequency band form $3.1 \mathrm{GHz}$ to $10 \mathrm{GHz}$ [1].

co its capability to coexist and share he spectrut consumption immunity to multipath fading interference mitiotion carrier free and the facility to overcome obstacles define this successful technology and differentiate it from traditional narrowband technologies [2]

Parallel to the increasing use of UWB technology, there has been a considerable interest in the mplementation of photonic solutions in order to profit the benefits of Microwave Photonics (MWP) [3]. For example, wireless transmission for UWB communications is able only to reach a distance of a few tens of meters. At this point, MWP leads to bring an elegant solution to this problem by increasing the coverage are through the distribution of the UWB signals in the optical domain. However, MWP is not only used to UWB signals distribution but also for UWB signal generation. Inherent advantages of the optical domain as low losses, light-weight, high bandwidth, tunability, reconfigurability and immunity to electromagnetic interference are useful in the process of the UWG signal generation

In this context, the proposal of techniques to generate UWB signals in the optical domain over traditional an pure electronic methods has been productive. We can find methods for UWB generation based on phasemodulation-to-intensity modulation conversion, photonic microwave filtering, and optical spectral shaping an dispersion-induced frequency to-time mapping [5]. Many schemes have been proposed to generate high order pulses in order to fulfil the FCC mask [4], however, the most usual signals that are being used in Impulse Radio UWB (IR-UWB) communications are monocycle and doublet pulses. For example, a simple method to general

In this paper, we present a scheme to generate UWB doublet pulses based on the use of nonlinearities present in an active integrated interferometric structure. The performance of the system exploits the properties of Mach-Zehnder interferometer (MZI) that integrates a semiconductor optical amplifier (SOA) in each branch [7]. An optical pulsed signal is used as pump signal in order to induce cross-phase modulation (XPM) in the SOA-MZI. The nonlinear characteristics of the SOA-MZI transfer function permits the generation of UWB doublet pulses for a given operation point which is determined by the saturation of the SOAs

\section{PRINCIPLE OF OPERATION}

Figure 1 shows the experimental scheme implemented by using an integrated SOA-MZI that contains two 1-mm length InGaAsP-InP SOAs with low polarization sensitivity. In principle, cross-gain modulation (XGM) and cross-phase modulation (XPM) processes could be present in the SOAs. However, the linewidth enhancement factor of the SOAs is large enough to neglect the XGM

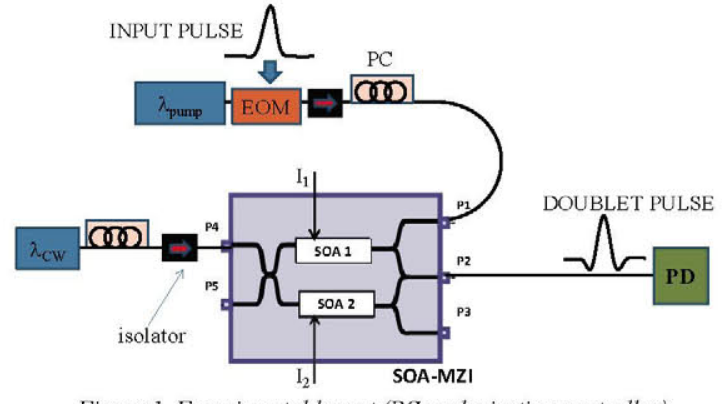

Figure 1. Experimental layout (PC: polarization controller).

The nonlinear SOA-MZI consists on an interferometric structure used in counter-propagation configuration with different input and output ports. In our case, a pulsed pump signal centred at the optical wavelength $\lambda_{\text {PUMP }}=1535 \mathrm{~nm}$ is obtained by external electrooptic modulation (EOM) of a CW optical carrier with an electrical signal (Gaussian pulse)

As shown in Fig. 1, the pump signal is introduced in the system at port P1 and a continuous probe wave CW) laser source is launched into Port P4 with a central optical wavelength $\lambda_{\mathrm{CW}}=1550 \mathrm{~nm}$. Thus, the conversion process is carricd out in counter-propagation configution, and hence, optical filtering is not needed to separate pump and probe signals. Finally, at the output port P2 the optical processing is performed and the optically processed signal is measured by means of a photodetector (PD) in an oscilloscope or an electrical

The process of XPM in the interferometric structure depends on the average optical power of the pump and probe signals and also it depends on the electrical currents applied to each SOA. Therefore, the optical power the pump and probe signals have to be controlled in order to optimize the conversion process. In Fig. 2, we represent tho consion tre lint bye modulating he pump signal with an RF the with a frequency or hase between the optical wavelength of the output port $\mathrm{P} 4$ and pump signal launched into port $\mathrm{Pl}$ depending on he determines the speed conversion of the probe continuous signal.
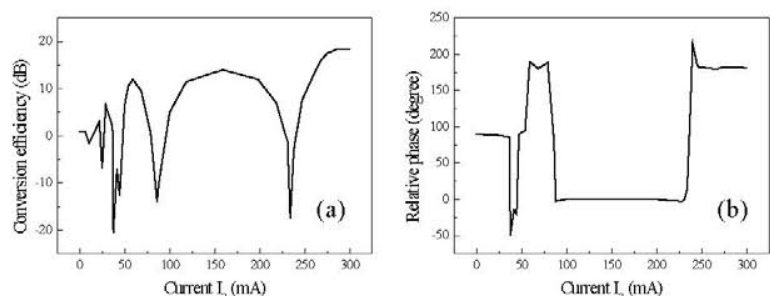

Figure 2. Transfer function of the SOA-MZI. Conversion amplitude (a) and relative phase (b) of the output por $P 2$ respect to input port $P 1$ in finction of the current applied to $S O A 2$.

We can observe that different operation current points can be achieved to perform a conventional wavelengt conversion. The maximum XPM conversion is found by applying to the SOA2 a current of $300 \mathrm{~mA}$. Since the transfer function is measured by means of a RF tone in low modulation regime, the maximum conversion corresponds to an operation point with a quasi-linear response while the points of minimum conversion correspond to a SOA-MZI transfer function with a quadratic response. In this way, the nonlinear response of the iransfer function can be achieved for currents far from maximum operation points in a similar way as in the proposal based on the nonlinear transfer function of an EOM [6]. In this case however, our system achieve higher extinction ratio and modulation efficiency than with ooventonal EOMs improving the performance of UWB pulse generation. In addition, we can observe also in Fig. 2 diferent regions where the relative phas between the output and input signal changes from $0^{\circ}$ to $180^{\circ}$. This change of sign leads to a control of the UWB pulse polarity in function of the applied current. 
3. EXPERIMENTAL RESULTS

In order to demonstrate the UWB generation approach, an experiment has been carried out according to Fig. 1. In this case, the pump laser is modulated with a quasi-Gaussian pulse train. The EOM is fed with an electrical signal coming from an electrical generator with a fixed pattern of 64 bits with one "1" and sixty-three " 0 " and of $80 \mathrm{ps}$ as shown in Fig. 3a. The optical output of the EOM corresponding to the pump signal is represented in Fig. $3 \mathrm{~b}$. We can observe that the EOM is biased in a negative region in order to obtain an inverted optical pulse. This is needed to guarantee the saturation of the SOA1 with high level of power coming from the pump signal.
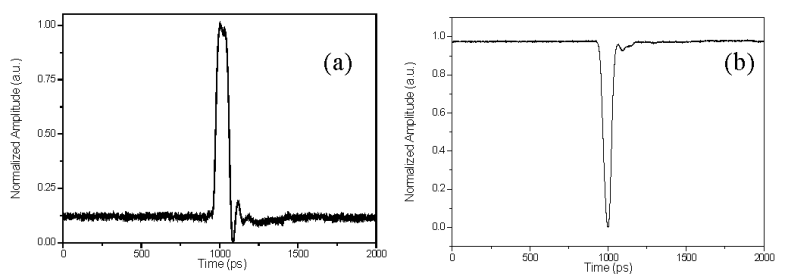

Figure 3: (a) Electrical pulsed signal and (b) optical signal at the output of EOM which is used as pump signal. As mentioned above, the pump signal is launched into the port P1 and the probe signal into the port P4. The doublet pulse is obtained at the output of the PD (port P2 in Fig. 1). In this case, we set the operation current of $\mathrm{A}$ and adjust the applied current to $\mathrm{SO} 2 \mathrm{A2}$ in order to monitor (ESA), respectively.

Firstly, a current of $300 \mathrm{~mA}$ is applied to SOA2 that corresponds to operation region in the transfer function with negative slope as plotted in Fig. 1. As expected, the output pulse is inverted leading to the generation of a quasi-Gaussian pulse as shown in Fig. 4a. The corresponding spectrum is plotted in Fig. 4b.
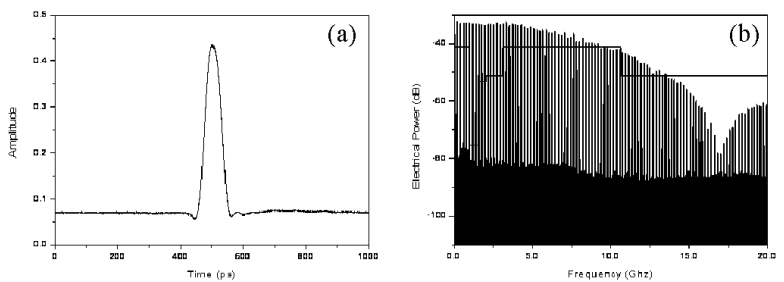

Figure 4: (a) Waveform and (b) electrical spectrum of the optical signal at the output of port $P 2$ when the current applied to SOA 2 is $300 \mathrm{~mA}$

When the electrical current is tuned to be a value of $270 \mathrm{~mA}$ in SOA2, the input pulse is large enough to aturate the SOA-MZT which permits to generate an UWB doublet as shown in the waveform of Fig. 5 a. As expected, the corresponding spectrum or Fig. Sb shows a redaction of spectral components close to baseband comparing with Fig. $4 \mathrm{~b}$. Also, the polarity of the UWB doublet pulse can be inverted by modifying the curren applied to SOA2. When SOA2 current is changed from $270 \mathrm{~mA}$ to $176 \mathrm{~mA}$, a UWB doublet with a reversed polarity is obtained. Figure $5 \mathrm{e}$ and $\mathrm{Fig}$. Sd represents the corresponding waveform and spectrum, respectively. to the fact that the conversion efficiency is lower for a current of $176 \mathrm{~mA}$ than $270 \mathrm{~mA}$ as shown in Fig. 2
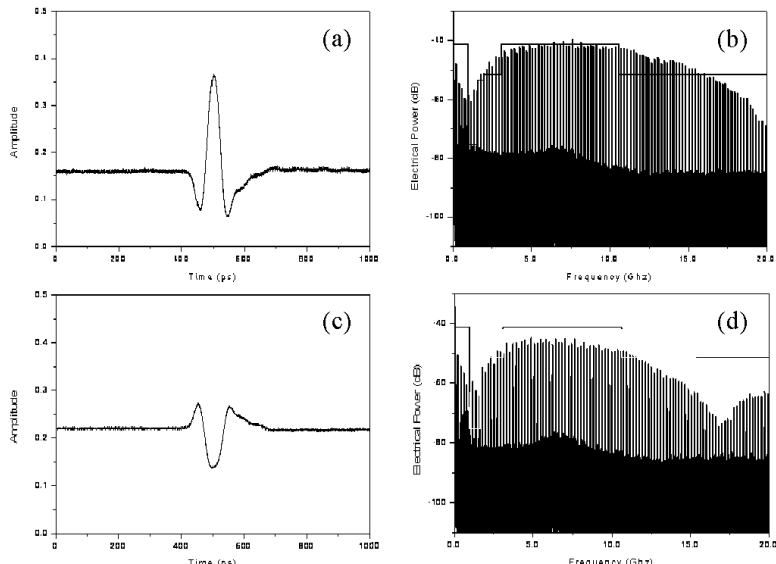

Figure 5: (a) Waveform of the UWB doublet pulse and (b) its corresponding electrical spectrum at the output port $P 2$ for an electrical current applied to SOA2 of $270 \mathrm{~mA}$. (c) Woveform and (d) its corrosponding spectrum of a UWB doublet pulse with inverted polarity for an electrical current applied to SOA2 of $176 \mathrm{~mA}$.

\section{CONCLUSIONS}

A novel approach has been proposed to generate UWB double pulses based on XPM in a SOA. An optical pulse is used as pump signal in order to convert a $\mathrm{CW}$ signal by means of XPM in an integrated SOA-MZT process which leads to the generation of UWB doublet pulses by means the epeation poin in the contersion SOA. Also, we have experimentally demonstroted the generation of UWB doublet pulses. Firstly, the biasing point of the SOA1 where the XPM is realized between the pump and probe signals is set to a maximum value 10 guarantee an optimum conversion. Then, the current of the SOA2 is changed to find a compromise between the conversion efficiency and the nonlinear response to obtain a UWB doublet pulse. In this way, the polarity of the doublet is controlled by switching the electrical current applied to SOA2

\section{ACKNOWLEDGEMENTS}

The research leading to these results has been funded by the national project TEC2010-21303-C04-02 and TEC2011-26642 funded by the

\section{REFERENCES}

11] D. Porcine, P. Research, W. Hirt, "Ultra-wideband radio technology: Potential and challenges ahead" IEEE Commum. Mag. 41, 66-74 (2003)

2] F. Nekoogar, Ultra-Wideband Communications: Fundamentals and Applications, Prentice Hall: United tates of America, (2006)

[3] J. Capmany, D. Novak, "Microwave photonics combines two worlds," Nature Photon. 1, 319-330 (2007).

J] J. Yao, F. Zeng, Q. Wang, "Photonic generation of ultrawideband signals," Journal of Lightwave Technology, vol. 25, no. 11, pp. 3219-3235, (2007).

[5] M. Bolea, J. Mora, B. Ortega, J. Capmany, High-order UWB pulse generation based on a microwave photonic filter using incoherent optical sources," In Proc. International Topical Meeting on Microwave Photonics Conference, pp. 462-465, (2011).

[6] Q. Wang, J. Yao, UWB doublet generation using nonlinearly-biased electro-optic intensity modulator," Electronics Letters, vol. 42, no. 22, pp. 1304,1305, Oct. 262006.

(C) negative coefficients using cross-phase modulation in an SOA-MZ interferometer," IEEE Photonics Techn Lett 20, 526-528 (2008). 